Article

\title{
On Thermomechanics of a Nonlinear Heat Conducting Suspension
}

\author{
Mehrdad Massoudi ${ }^{1, *,+}$ and A. D. Kirwan, Jr. ${ }^{2,+}$ \\ 1 U. S. Department of Energy, National Energy Technology Laboratory (NETL), 626 Cochrans Mill Road, \\ Pittsburgh, PA 15236, USA \\ 2 School of Marine Science and Policy, University of Delaware, 103 Robinson Hall, Newark, DE 19711, USA; \\ adk@udel.edu \\ * Correspondence: mehrdad.massoudi@netl.doe.gov; Tel.: +1-412-386-4975 \\ + These authors contributed equally to this work.
}

Academic Editor: Bekir S. Yilbas

Received: 10 March 2016; Accepted: 12 June 2016; Published: 18 June 2016

\begin{abstract}
In this short paper, we discuss and provide constitutive relations for the stress tensor and the heat flux vector for a nonlinear density-gradient dependent (Korteweg-type) fluid. Specifically, we attempt to present a unified thermo-mechanical approach to the two models given in papers of Massoudi (International Journal of Non-Linear Mechanics, 2001, 36(1), pp. 25-37.) and Massoudi (Mathematical Methods in the Applied Sciences, 2006, 29(13), pp. 1599-1613.) where the entropy law is used and restrictions are also obtained on the constitutive parameters. In most thermomechanical studies of nonlinear fluids using the entropy law, the stress tensor is assumed to be nonlinear and the heat flux vector still has the form of the Fourier type, i.e., it is proportional to the temperature gradient. In this paper, we use a generalized (nonlinear) form for the heat flux vector. When our model is linearized we obtain constraints, due to the entropy inequality, which are in agreement with the earlier results.
\end{abstract}

Keywords: heat flux vector; non-Fourier heat conduction; nonlinear fluids; continuum mechanics; Korteweg-type fluids; entropy inequality; Clausius-Duhem inequality

PACS: J0101

\section{Introduction}

Coal-water or coal-oil slurries are used as the primary fuel in many coal power plants. Studies have shown that the viscosity of coal-slurries depends on a few parameters such as the volume fraction of the coal particles, the mean size and the size distribution of the coal, and in some cases the shear rate ([1]). It has also been observed that preheating the fuel can result in better performance; as a result of such heating, the viscosity of the suspension may change. Constitutive modeling of nonlinear fluids (non-Newtonian fluids) has received much attention [2], where many empirical or semi-empirical equations have been suggested [3]. Most of these fluids are multi-component mixtures. Sometimes these fluids can be treated as a single continuum suspension with nonlinear material properties, and the techniques and models used in rheology or mechanics of nonlinear fluids can be used where global or macroscopic information about velocity or temperature fields for the whole suspension can be obtained. For some applications, however, there is a need to know the details of the field variables such as velocity, concentration, temperature, etc., and in these cases a multi-component modeling approach should be used. See [4-6] for examples of nonlinear fluids where both approaches can be used.

Density variations sometimes play a significant role in the theory of interacting continua, i.e., mixture theory, where constitutive relations are needed for the stress tensors, interaction forces, heat 
flux vectors, etc. Density gradients can appear as independent constitutive parameters in these theories. The "gradient-type" theories for fluids are well known in continuum mechanics, since the pioneering works of Stokes [7] and Maxwell [8]. The history of density-gradient dependent viscous fluids began with Korteweg in 1901, who modeled the effects of the fluid capillarity. He proposed a smooth constitutive equation for the stress tensor $[9,10]$,

$$
\mathbf{T}=(-p+\alpha \triangle \rho+\beta \nabla \rho \cdot \nabla \rho) \mathbf{I}+\gamma \nabla \rho \otimes \nabla \rho+\nu \nabla(\nabla \rho)+\lambda \operatorname{Tr}(\mathbf{D}) \mathbf{I}+2 \mu \mathbf{D}
$$

where $p, \alpha, \beta, \gamma, v, \lambda$ and $\mu$ are functions of $\rho$ and $\theta$, and $\triangle$ is the Laplacian operator. If we replace the density $\rho$ by the temperature $\theta$, we obtain an equation very similar to that given by Maxwell [8], who derived an equation for the stress tensor in rarified gases arising from inequalities of temperature. Dunn and Serrin ([11]) also studied the thermodynamics of density-gradient dependent viscous fluids. Later, Mehrabadi et al., [9] derived constitutive relations for a density-gradient dependent viscous fluid; they also discussed the relationship between their model and the Korteweg fluid (see also [12,13]). Density gradients may also play a role in the formulation of the constitutive relation. Rajagopal and Massoudi [14] derived a nonlinear expression for the Cauchy stress tensor which depends on the gradient of the density and the symmetric part of the velocity gradient. Their model has been generalized and used for a variety of applications [15,16]. In their model, Rajagopal and Massoudi [14] did not perform a second law analysis.

In most of the heat transfer problems, the classical theory of heat conduction proposed by Fourier ([17]; see also [18]) is used, where the heat flux is related to the temperature gradient

$$
\mathbf{q}=-k \nabla \theta
$$

where $k$ (thermal conductivity) is a material property and $\theta$ is the temperature. For complex materials, $k$ in the above equation is replaced with an effective or modified form of the thermal conductivity, which can depend on concentration, temperature, etc., and in fact, for anisotropic materials, $k$ becomes a second order tensor. For a discussion of the effective thermal conductivity concept in porous media and multiphase flows, see [19]. Massoudi [20,21], (without using a second law analysis) derived a constitutive relation for the heat flux vector, where $\mathbf{q}$ depends not only on the gradient of the temperature, but also on the gradient of the density and the symmetric part of the velocity gradient, $\mathbf{D}$.

In most thermomechanical studies of nonlinear fluids using the entropy law, the stress tensor is assumed to be nonlinear and the heat flux vector still has the form of the Fourier type, i.e., it is proportional to the temperature gradient. In this paper, we use a generalized (nonlinear) form for the heat flux vector. When our model is linearized we obtain constraints, due to the entropy inequality, which are in agreement with the earlier results. We present a unified thermo-mechanical approach to the two papers of Massoudi [20] and Massoudi and Mehrabadi [16], where the entropy law is used and restrictions are also obtained on the constitutive parameters. In Section 2, we discuss the governing equations, and in Section 3 we provide a short review of the two constitutive relations used in our study. In Section 4, which is the core of the current paper, we develop restrictions on the material properties obtained by applying the Clausius-Duhem inequality to the constitutive relations.

\section{The Governing Equations}

We assume that the nonlinear fluid can be modeled as a single continuum. The governing equations are the conservation of mass, the conservation of linear momentum, and the energy equation [22],

$$
\begin{aligned}
& \frac{\partial \rho}{\partial t}+\operatorname{div}(\rho \mathbf{v})=0 \\
& \rho \frac{d \mathbf{v}}{d t}=\operatorname{div} \mathbf{T}+\rho \mathbf{b}
\end{aligned}
$$




$$
\rho \frac{d \varepsilon}{d t}=\mathbf{T}: \mathbf{L}-\operatorname{div} \mathbf{q}+\rho r
$$

where $\mathbf{v}$ is the velocity, $\rho$ is the density, and $\frac{d}{d t}$ is the material time derivative, also shown as a superposed dot (), given by

$$
\frac{d()}{d t}=\frac{\partial()}{\partial t}+[\operatorname{grad}()] \mathbf{v}
$$

Here, $\mathbf{T}$ is the Cauchy stress tensor and $\mathbf{b}$ is the body force, $\varepsilon$ denotes the specific internal energy, $\mathbf{q}$ is the heat flux vector, $r$ is the radiant heating, and $\mathbf{L}$ is the velocity gradient. These are all identified in the nomenclature list at the beginning of the paper. The balance of angular momentum indicates that in the absence of couple stresses the Cauchy stress tensor is symmetric. In continuum mechanics applications, thermodynamical considerations [10,23-25] require the application of the second law of thermodynamics or the entropy inequality. The local form of the entropy inequality is given by [25]:

$$
\rho \dot{\eta}+\operatorname{div} \varphi-\rho s \geq 0
$$

where $\eta(\mathbf{x}, t)$ is the specific entropy density, $\varphi(\mathbf{x}, t)$ is the entropy flux, and $s$ is the entropy supply density due to external sources. Rajagopal and colleagues (see for example, [26,27]) have devised a thermodynamic framework by appealing to the maximization of the rate of entropy. This is different from the traditional thermodynamic approach (for example, Dunn and Fosdick [28]) whereby a form for the stress is assumed (or derived) and restrictions on the material parameters are obtained by invoking the Clausius-Duhem inequality. In this new thermodynamic approach, specific forms for the Helmholtz potential and the rate of dissipation are assumed at the outset.

Assuming that $\varphi=\theta^{-1} \mathbf{q}$ and $s=\theta^{-1} r$ where $\theta$ is the absolute temperature, (7) is reduced to the Clausius-Duhem inequality

$$
\rho \dot{\eta}+\operatorname{div} \frac{\mathbf{q}}{\theta}-\rho \frac{r}{\theta} \geq 0
$$

For a detailed discussion of the Clausius-Duhem inequality, we refer the reader to [25]. For a detailed discussion of the above equations as given within the context of Rational Thermodynamics, we refer the reader to [29].

Inspection of the governing equations reveals that constitutive equations are needed for $\mathbf{T}, \mathbf{q}, \varepsilon$, and $r$. In this paper, we ignore the effects of radiation, and we do not directly discuss or assume any particular form for the constitutive relation for $\varepsilon$. The emphasis here is on constitutive modeling of $\mathbf{T}$ and $\mathbf{q}$ for potential application to nonlinear suspensions such as coal-slurries.

\section{The Constitutive Equations}

The most important constitutive relations in the thermomechanics of fluids are the (Cauchy) stress tensor $\mathbf{T}$ and the heat flux vector $\mathbf{q}$ (related to heat conduction). Since the publication of the paper by Rivlin and Ericksen [30], where fluids of complexity $n$ were discussed [10], to a recently published book [31], the term complex fluids refers to fluid-like materials whose response functions, namely the stress tensor and the heat flux vector, are "nonlinear" in some fashion.

\subsection{Stress Tensor}

In this paper, we study a fluid-like material where the density is a variable. In fluid suspensions, the volume fraction field $\phi(\mathbf{x}, t)$ plays a major role; this is a continuous function of position and time ([32]) and is an independent kinematical variable called the volume distribution function with the property $0 \leq \phi(\mathbf{x}, t) \leq \phi_{\max } \leq 1$. Before the process of homogenization, $\phi$ is either one or zero at any position and time. The classical mass density or bulk density $\rho$ is related to $\rho_{s}$ and $\phi$ through $\rho=\rho_{s} \phi$, where $\rho_{s}$ is the density of a single particle. It is clear that in practice $\phi$ is never equal to one: it has a maximum value, known as the maximum packing fraction. 
We start with the constitutive relation derived by Rajagopal and Massoudi [14], which was generalized and elaborated by [16], where the Cauchy stress tensor depends on the gradient of the density (or volume fraction) and the symmetric part of the velocity gradient. For a detailed description of this model, the reader is referred to [15]. In the original work by Rajagopal and Massoudi [14], they assumed: $\beta_{0}(\rho)=\kappa \rho ; \beta_{1}(\rho)=\beta_{10}+\beta_{11} \rho+\beta_{12} \rho^{2} ; \beta_{2}(\rho)=\beta_{20}+\beta_{21} \rho+\beta_{22} \rho^{2}$ and $\beta_{3}(\rho)=\beta_{30}+\beta_{31} \rho+\beta_{32} \rho^{2} ; \beta_{4}(\rho)=\beta_{40}+\beta_{41} \rho+\beta_{42} \rho^{2}$ where in general, the material parameters $\kappa, \beta^{\prime}$ s have to be measured experimentally. In [14], no couple stresses or temperature variations were allowed. Nevertheless, due to the presence of higher order gradient terms, it is necessary to provide additional boundary conditions before one can solve practical and simple boundary value problems. These issues are discussed in [16].

They [16], that is, Massoudi and Mehrabadi, assumed that the stress tensor $\mathbf{T}$ can be expressed as $\mathbf{T}=\mathbf{T}(\rho, \operatorname{grad} \rho, \mathbf{D})$, and using representation theorems (see [33]), the following form is obtained:

$$
\mathbf{T}=\left[\beta_{0}(\rho)+\beta_{1}(\rho) \nabla \rho \cdot \nabla \rho+\beta_{2}(\rho) \operatorname{tr} \mathbf{D}\right] \mathbf{I}+\beta_{3}(\rho) \mathbf{D}+\beta_{4} \nabla \rho \otimes \nabla \rho+\beta_{5}(\rho) \mathbf{D}^{2}
$$

where

$$
\begin{aligned}
& \beta_{0}=f \rho \\
& \beta_{1}=\beta_{1}^{*}\left(1+\rho+\rho^{2}\right) \\
& \beta_{2}=\beta_{2}^{*}\left(1+\rho+\rho^{2}\right) \\
& \beta_{3}=\beta_{3}^{*}\left(1+\rho+\rho^{2}\right) \\
& \beta_{4}=\beta_{4}^{*}\left(1+\rho+\rho^{2}\right) \\
& \beta_{5}=\beta_{5}^{*}\left(1+\rho+\rho^{2}\right)
\end{aligned}
$$

In (9), $\otimes$ designates the outer (dyadic) product of two vectors, $t r$ is the trace of a second order tensor, and $\mathbf{I}$ is the identity tensor. The coefficients $\beta_{i}$ are material properties which are assumed to be functions of the density (or volume fraction) and $\mathbf{D}=\frac{1}{2}\left[\nabla \mathbf{v}+(\nabla \mathbf{v})^{T}\right]$. We can think of the above representation as a Taylor series approximation for the material parameters [34]. Rajagopal \& Massoudi [14] provided the following interpretations: $\beta_{0}(\rho)$ is similar to the pressure in a compressible fluid where an equation of state is needed, $\beta_{2}(\rho)$ is similar to the second coefficient of viscosity in a compressible fluid, $\beta_{1}(\rho)$ and $\beta_{4}(\rho)$ are the material parameters related to the distribution of the particles, $\beta_{3}(\rho)$ is the viscosity, and $\beta_{5}(\rho)$ is the coefficient of cross viscosity. Rajagopal et al. [35] proved existence of solutions, in case of the fully developed flow down an incline plane for a selected range of parameters, when

$$
\begin{aligned}
& \beta_{1}+\beta_{4} \geq 0 \\
& f<0
\end{aligned}
$$

Now, (9) can also be written as

$$
\mathbf{T}=\mathbf{T}_{\mathbf{e}}+\mathbf{T}_{\mathbf{d}}
$$

where

$$
\mathbf{T}_{e}=\left[\beta_{0}(\rho)+\beta_{1}(\rho) \nabla \rho \cdot \nabla \rho\right] \mathbf{I}+\beta_{4}(\rho) \nabla \rho \otimes \nabla \rho
$$

$\mathbf{T}_{e}$ can be thought of as the equilibrium part of the stress tensor and

$$
\mathbf{T}_{d}=\left[\beta_{2}(\rho) \operatorname{tr} \mathbf{D}\right] \mathbf{I}+\beta_{3}(\rho) \mathbf{D}+\beta_{5}(\rho) \mathbf{D}^{2}
$$

$\mathbf{T}_{d}$ is the dynamic contribution. 
Equation (9) can also represent a constitutive model for a fluid which is capable of exhibiting normal stress effects. For example, if $\beta_{1}=\beta_{2}=\beta_{4}=0$, then the model reduces to a form similar to the Reiner-Rivlin fluid model.

\subsection{Heat Flux Vector}

Fourier, in 1822, proposed a constitutive relationship for the heat flux vector, where

$$
\mathbf{q}=-k \nabla \theta
$$

$\mathbf{q}$ is the heat flux vector, $\theta$ is the temperature, $\nabla$ is the gradient operator, and $k$ is a material property known as thermal conductivity. It is interesting to note that Fourier considered the thermal conductivity of a material as a mathematical concept rather than an experimentally measurable parameter (see [36]). In heat transfer studies related to porous media, an effective thermal conductivity is usually used (see [19]); several studies have shown that in some cases the heat flux vector should depend not only on the temperature gradient but also on the shear rate, and other parameters.

Massoudi [20,21], derived a general nonlinear constitutive relation for $\mathbf{q}$ (see Section 3 of [20]) with no entropy analysis. There, it is assumed that the heat flux vector $\mathbf{q}$ can be expressed as $\mathbf{q}=\mathbf{q}(\theta, \operatorname{grad} \theta, \rho, \operatorname{grad} \rho, \mathbf{D})$, and using representation theorems (see [33]), the following form is obtained:

$$
\mathbf{q}=a_{1} \mathbf{n}+a_{2} \mathbf{m}+a_{3} \mathbf{D n}+a_{4} \mathbf{D m}+a_{5} \mathbf{D}^{2} \mathbf{n}+a_{6} \mathbf{D}^{2} \mathbf{m}
$$

where $a_{1}=-k$ (thermal conductivity) and $a_{2}-a_{6}$ are scalar functions of

$$
\begin{aligned}
& \rho, \theta, \mathbf{m} \cdot \mathbf{m}, \mathbf{n} . \mathbf{n}, \mathbf{m} \cdot \mathbf{n}, I_{1}=\operatorname{tr} \mathbf{D}, I_{2}=\operatorname{tr} \mathbf{D}^{2}, I_{3}=\operatorname{tr} \mathbf{D}^{3} \\
& \text { n.Dn, } \mathbf{n} \cdot \mathbf{D}^{\mathbf{2}} \mathbf{n}, \mathbf{m} \cdot \mathbf{D m}, \mathbf{m} \cdot \mathbf{D}^{2} \mathbf{m}, \mathbf{n} \cdot \mathbf{D m}, \mathbf{n} \cdot \mathbf{D}^{2} \mathbf{m}
\end{aligned}
$$

In this formulation, $\mathbf{m}=\operatorname{grad} \rho$ and $\mathbf{n}=\operatorname{grad} \theta$. Without a thermodynamic analysis, it is not clear whether the material properties (coefficients) $a_{i}$ are positive, negative, or zero. An equivalent version of (16) appropriate for a second law analysis is

$$
\mathbf{q}=\left(a_{1} \mathbf{I}+a_{3} \mathbf{D}+a_{5} \mathbf{D}^{2}\right) \nabla \theta+\left(a_{2} \mathbf{I}+a_{4} \mathbf{D}+a_{6} \mathbf{D}^{2}\right) \nabla \rho
$$

It is noted that similar expressions were obtained by Wang [37] and Jaric and Golubovic [38].

We can see that if $a_{2}=a_{3}=a_{4}=a_{5}=a_{6}=0$, then Equation (16) or (18) reduces to the classical heat conduction law due to Fourier. Yang et al. [39] used Equation (16) to study the heat transfer and the flow of a nonlinear fluid flowing down an inclined plane. They non-dimensionalized the resulting equations and performed a parametric study. In their problem, due to the simplifying kinematical assumptions, only the effects of terms multiplied by $a_{1}, a_{2}, a_{5}$, and $a_{6}$ were included. However, other than $a_{1}$ (which is related to the thermal conductivity of the fluid), it was not clear whether the dimensionless numbers arising from $a_{2}, a_{5}$, and $a_{6}\left(P_{2}, P_{5}\right.$, and $P_{6}$ in their paper) were positive or negative. Therefore, in their numerical simulations they considered both the positive and negative cases for these dimensionless numbers and as a result very interesting temperature profiles were obtained. They, however, raised the question that some of these solutions might not be physically realistic due to the uncertainty in the sign of these material parameters. It therefore became necessary to do a thermodynamic (entropy) analysis for the constitutive relation given by (16). This is considered next.

\section{Entropy Analysis}

Entropy analyses of granular type constitutive models are complicated by the fact that they generally are incompatible with conventional notions of thermodynamics unless the interaction coefficients are severely restricted as noted in [11]. Nevertheless, such an analysis is important. 
As discussed by Hutter and Schneider [40], both objectivity and second law analyses reduce constitutive equations to a "minimal" form. See also [41,42] for additional discussion regarding objectivity and constraints imposed by the second law.

A useful approach to overcome this matter is to postulate additional kinematic terms that appear in the energy equation to accommodate interactions between the density and velocity fields. For example, Goodman and Cowin [32] introduce a dissipative "intrinsic equilibrated body force" while Dunn and Serrin [11] introduce an "interstitial work flux." These appear in the energy equation and hence the entropy inequality. Both studies provide an entropy analysis and obtain constraints on phenomenological coefficients consistent with linear viscous fluids and Fourier's law for heat conduction. However, there are additional constraints arising from the inclusion of the additional terms in the entropy inequality. The Goodman and Cowin [32] study relies on both the phenomenological coefficients and the volume fraction, while in Dunn and Serrin [11] the inequality involves the phenomenological coefficients and the density gradient and temperature.

The objective of the current work is to present a unified thermo-mechanical approach to the models given in the two papers of Massoudi [20] and Massoudi \& Mehrabadi [16]. We will attempt to use the entropy law to obtain restrictions on the constitutive equations. Our starting point is the theory given by $[15,16]$, where a general nonlinear constitutive relation is derived for $\mathbf{T}$ (with no entropy analysis). A more general case is included in the papers by Massoudi [20] and Mehrabadi et al. [9], where a general nonlinear constitutive relation is derived for $q$ (with no entropy analysis). Starting with $\mathbf{T}$ as given in (9) we have:

$$
\mathbf{T}(\rho, \theta, \operatorname{grad} \theta, \mathbf{D})=\left[\beta_{0}(\rho)+\beta_{1}(\rho) \nabla \rho+\beta_{2}(\rho) \operatorname{tr} \mathbf{D}\right] \mathbf{I}+\beta_{3}(\rho) \mathbf{D}+\beta_{4}(\rho) \nabla \rho \otimes \nabla \rho
$$

And for q:

$$
\mathbf{q}(\rho, \theta, \operatorname{grad} \theta, \mathbf{D})=\left(a_{1} \mathbf{I}+a_{3} \mathbf{D}\right) \nabla \theta+\left(a_{2} \mathbf{I}+a_{4} \mathbf{D}\right) \nabla \rho
$$

Note that for the analysis here the nonlinear terms in $\mathbf{D}$ are neglected. That is, in (8) and (15) $\beta_{5}=a_{5}=a_{6}=0$. Furthermore, eliminating $\rho r$ between Equations (5) and (7) and introducing the Helmholtz function $\psi=\epsilon-\theta \eta$ gives the basic dissipation inequality

$$
-\rho(\dot{\psi}+\eta \dot{\theta})+\mathbf{T}: \mathbf{L}-(\mathbf{q} / \theta) \cdot \nabla \theta \geq 0
$$

Inserting (19) and (20) into (21) gives

$$
\begin{aligned}
& {\left[\beta_{0} \operatorname{tr} \mathbf{D}+\beta_{1}|\nabla \rho|^{2} \operatorname{tr} \mathbf{D}+\beta_{2}(\operatorname{tr} \mathbf{D})^{2}\right]+\beta_{3} \mathbf{D}: \mathbf{D}+\beta_{4}(\nabla \rho \otimes \nabla \rho): \mathbf{D}-a_{1}|\nabla \theta|^{2}} \\
& -a_{2}(\nabla \rho \cdot \nabla \theta)-a_{3} \nabla \theta \cdot(\mathbf{D} \nabla \theta)-a_{4}(\mathbf{D} \nabla \rho) \cdot \nabla \theta-\rho(\dot{\psi}+\eta \dot{\theta}) \geq 0
\end{aligned}
$$

Clearly

$$
\begin{aligned}
\beta_{3} & \geq 0 \\
\beta_{2}+\beta_{3} / 3 & \geq 0 \\
a_{1} & \leq 0
\end{aligned}
$$

The remaining coefficients are constrained by

$$
\begin{aligned}
& \beta_{3} \mathbf{D}: \mathbf{D}+\beta_{2}(\operatorname{tr} \mathbf{D})^{2}-a_{1}|\nabla \theta|^{2}+\beta_{0} \operatorname{tr} \mathbf{D}+\beta_{1}|\nabla \rho|^{2} \operatorname{tr} \mathbf{D}+\beta_{4}(\nabla \rho \otimes \nabla \rho): \mathbf{D}-a_{2}(\nabla \rho \cdot \nabla \rho) \\
& -a_{3} \nabla \theta \cdot(\mathbf{D} \nabla \theta)-a_{4}(\mathbf{D} \nabla \rho) \cdot \nabla \theta-\rho(\dot{\psi}+\eta \dot{\theta}) \geq 0
\end{aligned}
$$


For comparison with other results it is useful to replace the tensors $\mathbf{D}$ and $\nabla \rho \otimes \nabla \rho$ by their trace and deviatoric components, i.e., $\mathbf{D}=\hat{\mathbf{D}}+\mathbf{I} D / 3$ and $\nabla \rho \otimes \nabla \rho=\hat{\mathbf{M}}+|\nabla \rho|^{2} / 3$. Then (24) becomes

$$
\begin{aligned}
& \beta_{3} \hat{\mathbf{D}}^{2}+\left(\beta_{2}+\beta_{3} / 3\right) D^{2}+\beta_{4} \hat{\mathbf{D}}: \hat{\mathbf{M}}+\left(\beta_{1}+\beta_{4} / 3\right) D|\nabla \rho|^{2}+\beta_{0} D-a_{2}(\nabla \rho \cdot \nabla \theta) \\
& -a_{3}(\mathbf{D} \nabla \theta) \cdot \nabla \theta-a_{4}(\mathbf{D} \nabla \rho) \cdot \nabla \rho-\rho(\dot{\psi}+\eta \dot{\theta}) \geq 0
\end{aligned}
$$

(where $D=\operatorname{tr} \mathbf{D}$ ).

In the Dunn and Serrin [28] theory, $\mathbf{T}$ does not depend on $\nabla \rho$ and $\mathbf{q}$ depends only on $\nabla \theta$. That is, $\beta_{1}=\beta_{4}=a_{2}=a_{3}=a_{4}=0$ and $\beta_{0} \operatorname{tr} \mathbf{D}$ were replaced by the "interstitial working" and a constitutive equation for $\psi$. The inequality (25) is then in agreement with the results of Massoudi \& Mehrabadi for a Korteweg material.

The general condition proposed here admits a special case that is more general than their theory. If one imposes an equation of state $\rho=\rho(\theta)$ and $\partial \rho / \partial \theta=r$, then (25) simplifies to

$$
\begin{aligned}
& \beta_{3} \mathbf{D}: \mathbf{D}+\beta_{2}(t r \mathbf{D})^{2}-a_{1}|\nabla \theta|^{2}+\beta_{0} t r \mathbf{D}+r^{2} \beta_{1}|\nabla \theta|^{2} t r \mathbf{D}+r^{2} \beta_{4}(\nabla \theta \otimes \nabla \theta): \mathbf{D}= \\
& a_{2} r|\nabla \theta|^{2}-a_{3} \nabla \theta \cdot(\mathbf{D} \nabla \theta)-a_{4} r(\mathbf{D} \nabla \theta) \cdot \nabla \theta-\rho(\dot{\psi}+\eta \dot{\theta}) \geq 0
\end{aligned}
$$

Now recall the existence criteria of Rajagopal and Massoudi [14] and Rajagopal et al. [35], $\beta_{1}+\beta_{4}>0$ and $a_{1}<0$. The latter criterion is consistent with (26), i.e., the heat flux is consistent with the second law inequality, (7). The former criterion suggests that for solutions to exist the density and strain rate fields must be consistently aligned. Intuition suggests that $\hat{\mathbf{D}}: \hat{\mathbf{M}}>0$. This implies $\beta_{4} \geq 0$ for entropy generation. The existence criterion requires that $\beta_{1}>-\beta_{4}$. This is consistent with (23) for incompressible material. However, for compressible material, the second law inequality and the existence criterion imply a nuanced relation between the magnitude of the density gradient and the divergence of the velocity field.

\section{Discussion and Conclusions}

The textbook examples of entropy analysis of materials focus on linear constitutive equations. Then the entropy production terms are quadratic in the objective constitutive variables and entropy analysis is straightforward. Entropy production lies on a quadratic surface in the phase space defined by the constitutive variables. Non-negativity (or convexity of the surface) is assured by appropriate constraints on the phenomenological parameters. We are unaware of comparable entropy analyses involving nonlinear constitutive equations.

As shown here, the production terms do not reduce to simple quadratic forms as is the case for linear viscous fluids and heat conducting materials. Instead, they form complicated surfaces in the constitutive variable phase space. Unlike quadratic surfaces, there is no simple paradigm for ensuring convexity. Depending on solution properties, positive entropy production may be restricted to portions of this phase space. How might one find this region? Rajagopal et al. [14,35] showed that the existence of solutions required constraints on some of the phenomenological coefficients. Here we showed that these were consistent with the entropy analysis constraints. This suggests a fundamental connection between existence requirements and positive entropy production.

Acknowledgments: We thank Karal Gregory for technical assistance with the manuscript.

Author Contributions: Mehrdad Massoudi and A. D. Kirwan conceived and formulated the problem; Mehrdad Massoudi and A. D. Kirwan both contributed to the derivation, the writing, the editing of the paper. A. D. Kirwan performed the analysis in Section 4.

Conflicts of Interest: The authors declare no conflict of interest. 


\section{Nomenclature}

D symmetric part of the velocity gradient

I identity tensor

L gradient of the velocity vector

T Cauchy stress tensor

$a_{i} \quad$ heat flux vector constitutive coefficients, $i=1$ to 6

b body force vector

div divergence operator

$k$ thermal conductivity

q heat flux vector

$r \quad$ radiant heating

u velocity vector

$\mathbf{x} \quad$ spatial position

$\beta_{i} \quad$ material constitutive coefficients, $i=0$ to 5

$\varepsilon \quad$ specific internal energy

$\theta \quad$ temperature

$\rho \quad$ bulk density

$\rho_{10} \quad$ reference density

$\phi \quad$ volume fraction

$\nabla \quad$ gradient symbol

$\triangle \quad$ Laplacian operator

$\otimes \quad$ outer product

\section{References}

1. Gupta, G.; Massoudi, M. Flow of a generalized second grade fluid between heated plates. Acta Mech. 1993, 99, 21-33.

2. Schowalter, W.R. Mechanics of Non-Newtonian Fluids; Pergamon Press: New York, NY, USA, 1978.

3. Massoudi, M.; Vaidya, A. On some generalizations of the second grade fluid model. Nonlinear Anal. Real World Appl. 2008, 9, 1169-1183.

4. Rajagopal, K.R.; Tao, L. Mechanics of Mixtures; World Scientific: Singapore, 1995.

5. Massoudi, M. A note on the meaning of mixture viscosity using the classical continuum theories of mixtures. Int. J. Eng. Sci. 2008, 46, 677-689.

6. Massoudi, M. A Mixture Theory formulation for hydraulic or pneumatic transport of solid particles. Int. J. Eng. Sci. 2010, 48, 1440-1461.

7. Stokes, G.G. On the theories of the internal friction of fluids in motion, and of the equilibrium and motion of elastic solids. Trans. Camb. Philos. Soc. 1845, 8, 287-305.

8. Maxwell, J.C. On stresses in rarified gases arising from inequalities of temperature. Philos. Trans. R. Soc. Lond. 1876, 170, 231-256.

9. Mehrabadi, M.M.; Cowin, S.C.; Massoudi, M. Conservation laws and constitutive relations for density-gradientdependent viscous fluids. Contin. Mech. Thermodyn. 2005, 17, 183-200.

10. Truesdell, C.; Noll, W. The Non-Linear Field Theories of Mechanics; Springer Verlag: New York, NY, USA, 1992.

11. Dunn, J.E.; Serrin, J. On thermodynamics of interstitial working. Arch. Ration. Mech. Anal. 1985, 88, 95-133.

12. Blinowski, A. On the order of magnitude of the gradient-of-density dependent part of an elastic potential in liquids. Arch. Mech. 1973, 25, 833-849.

13. Blinowski, A. Gradient description of capillary phenomena in multicomponent fluids. Arch. Mech. 1975, 27, 273-292.

14. Rajagopal, K.R.; Massoudi, M. A Method for Measuring Material Moduli of Granular Materials: Flow in an Orthogonal Rheometer; Topical Report DOE/PETC/TR-90/3; USDOE Pittsburgh Energy Technology Center: Pittsburgh, PA, USA, 1990. 
15. Massoudi, M. On the flow of granular materials with variable material properties. Int. J. Non-Linear Mech. 2001, 36, 25-37.

16. Massoudi, M.; Mehrabadi, M.M. A continuum model for granular materials: Considering dilatancy, and the Mohr-Coulomb criterion. Acta Mech. 2001, 152, 121-138.

17. Fourier, J. The Analytical Theory of Heat; Freeman, A., Translator; Dover Publications, Inc.: Mineola, NY, USA, 1955.

18. Winterton, R.H.S. Heat in history. Heat Transf. Eng. 2001, 22, 3-11.

19. Kaviany, M. Principles of Heat Transfer in Porous Media, 2nd ed.; Springer Verlag: New York, NY, USA, 1995.

20. Massoudi, M. On the heat flux vector for flowing granular materials. Part 2: Derivation and special cases. Math. Methods Appl. Sci. 2006, 29, 1599-1613.

21. Massoudi, M. On the heat flux vector for flowing granular materials. Part 1: Effective thermal conductivity and background. Math. Methods Appl. Sci. 2006, 29, 1585-1598.

22. Slattery, J.C. Advanced Transport Phenomena; Cambridge University Press: Cambridge, UK, 1999.

23. Müller, I.A. On the entropy inequality. Arch. Ration. Mech. Anal. 1967, 26, 118-141.

24. Ziegler, H. An Introduction to Thermomechanics, 2nd ed.; North-Holland: New York, NY, USA, 1983.

25. Liu, I.S. Continuum Mechanics; Springer Verlag: Berlin, Germany, 2002.

26. Rajagopal, K.R.; Srinivasa, A.R. A thermodynamic frame work for rate type fluid models. J. Non-Newton. Fluid Mech. 2000, 88, 207-227.

27. Rajagopal, K.R.; Srinivasa, A.R. Modeling anisotropic fluids within the framework of bodies with multiple natural configurations. J. Non-Newton. Fluid Mech. 2001, 99, 109-124.

28. Dunn, J.E.; Fosdick, R.L. Thermodynamics, stability, and boundedness of fluids of complexity 2 and fluids of second grade. Arch. Ration. Mech. Anal. 1974, 56, 191-252.

29. Truesdell, C. Rational Thermodynamics, 2nd ed.; Springer Verlag: New York, NY, USA, 1984.

30. Rivlin, R.S.; Ericksen, J.L. Stress-deformation relations for isotropic materials. J. Ration. Mech. Anal. 1955, 4, 323-425.

31. Deshpande, A.P.; Krishnan, J.M.; Kumar, S., Eds. Rheology of Complex Fluids; Science and Business Media, Springer: Berlin, Germany, 2010.

32. Goodman, M.A.; Cowin, S.C. Continuum Theory for Granular Materials. Arch. Ration. Mech. Anal. 1972, 44, 249-266.

33. Spencer, A.J.M. Theory of invariants. In Continuum Physics; Eringen, A.C., Ed.; Academic Press: Cambridge, MA, USA, 1971; Part III, Volume 1, pp. 239-353.

34. Walton, O.R.; Braun, R.L. Stress calculations for assemblies of inelastic spheres in uniform shear. Acta Mech. 1986, 63, 73-86.

35. Rajagopal, K.R.; Troy, W.C.; Massoudi, M. Existence of solutions to the equations governing the flow of granular materials. Eur. J. Mech. B Fluids 1992, 11, 265-276.

36. Narasimhan, T.N. Fourier's heat conduction equation: History, influence, and connections. Rev. Geophys. 1999, 37, doi:10.1029/1998RG900006.

37. Wang, L. Vector-field theory of heat flux in confective heat transfer. Nonlinear Anal. 2001, 47, 5009-5020.

38. Jaric, J.; Golubovic, Z. Fourier's law of heat conduction in a non-linear fluid. J. Therm. Stresses 1999, 22, 293-303.

39. Yang, H.; Aubry, N.; Massoudi, M. Heat transfer in granular materials: Effects of nonlinear heat conduction and viscous dissipation. Math. Methods Appl. Sci. 2013, 36, 1947-1964.

40. Hutter, K.; Schneider, L. Important aspects in the formulation of solid-fluid debris-flow models. Part I. Thermodynamic implications. Contin. Mech. Thermodyn. 2010, 22, 363-390.

41. Hutter, K.; Rajagopal, K.R. On flows of granular material. Contin. Mech. Thermodyn. 1994, 6, 81-139.

42. Kirwan, A.D., Jr. On Objectivity, Irreversibility, and Non-Newtonian Fluids. Fluids 2016, 1, 3.

(C) 2016 by the authors; licensee MDPI, Basel, Switzerland. This article is an open access article distributed under the terms and conditions of the Creative Commons Attribution (CC-BY) license (http://creativecommons.org/licenses/by/4.0/). 